\title{
Die 57. Internationale Mathematik-Olympiade
}

\author{
Jürgen Prestin
}

Die 57. Internationale Mathematik-Olympiade (IMO) fand vom 6.-16. Juli 2016 mit 7I Schülerinnen (1I,8\%) und 53I Schülern aus 109 Ländern in Hongkong statt. Damit wurde ein neuer Teilnehmerrekord aufgestellt.

Die deutsche Mannschaft bestand aus sechs Schülerinnen und Schülern (Tabelle I), Dr. Eric Müller als stellvertretendem Delegationsleiter und dem Berichterstatter als Delegationsleiter.

Alle sechs Teilnehmer haben schon große Erfolge bei nationalen Wettbewerben erringen können. Sie nahmen an allen Bundesrunden der Mathematik-Olympiade seit 2013 teil, Sebastian Meyer, Manfred Paul und Ferdinand Wagner sogar schon seit 20I2. Alle erhielten auch einen Preis bei der 2. Runde des Bundeswettbewerbs Mathematik 2015. Branko Juran ist Bundessieger 2014 und 20I5, Ferdinand Wagner sogar viermaliger Bundessieger seit 2012. Martin Drees gewann als Zwölfjähriger die Weltmeisterschaft im Kopfrechnen der Kinder.

Tabelle I. Das deutsche Team

\begin{tabular}{|c|c|c|c|}
\hline Name & Wohnort & Schule & Klasse \\
\hline $\begin{array}{l}\text { Armbruster, } \\
\text { Susanne }\end{array}$ & Unterhaching & $\begin{array}{l}\text { Lise-Meitner-Gymnasium } \\
\text { Unterhaching }\end{array}$ & 12 \\
\hline $\begin{array}{l}\text { Drees, } \\
\text { Martin }\end{array}$ & Cadolzburg & $\begin{array}{l}\text { Dürer-Gymnasium } \\
\text { Nürnberg }\end{array}$ & II \\
\hline $\begin{array}{l}\text { Juran, } \\
\text { Branko }\end{array}$ & Berlin & $\begin{array}{l}\text { Heinrich-Hertz- } \\
\text { Oberschule Berlin }\end{array}$ & II \\
\hline $\begin{array}{l}\text { Meyer, } \\
\text { Sebastian }\end{array}$ & Dresden & $\begin{array}{l}\text { Martin-Andersen-Nexö- } \\
\text { Gymnasium Dresden }\end{array}$ & II \\
\hline $\begin{array}{l}\text { Paul, } \\
\text { Manfred }\end{array}$ & Rimpar & $\begin{array}{l}\text { Deutschhaus- } \\
\text { Gymnasium Würzburg }\end{array}$ & 11 \\
\hline $\begin{array}{l}\text { Wagner, } \\
\text { Ferdinand }\end{array}$ & Leipzig & $\begin{array}{l}\text { Friedrich-Schiller- } \\
\text { Gymnasium Leipzig }\end{array}$ & 12 \\
\hline
\end{tabular}

I Auswahl und Vorbereitung der deutschen Mannschaft

Die Auswahl und Vorbereitung des deutschen Teams verlief nach dem Verfahren der Vorjahre. Es qualifizierten sich 96 Schüler und 17 Schülerinnen durch die erfolgreiche Teilnahme an der 2. Runde des Bundeswettbewerbs Mathematik oder an der Bundesrunde der MathematikOlympiaden für zwei Auswahlklausuren am 2. und 9. Dezember 20I5. Hieran nahmen 87 Schüler und 16 Schülerinnen teil. Die 16 erfolgreichsten Klausurteilnehmer bildeten den Kandidatenkreis für das deutsche Team. Für diese I 5 Schüler und eine Schülerin gab es Seminare über eine knappe Woche in Rostock, drei Wochenenden in Bad Homburg (jeweils 3 Tage) und die traditionelle Abschlusswoche am Mathematischen Forschungsinstitut in Oberwolfach. Während dieser Zeit wurden von allen Kandidaten insgesamt sieben Klausuren geschrieben. Die sechs Besten qualifizierten sich für das IMO-Team, dessen Zusammensetzung am 26. Mai in Oberwolfach verkündet wurde.

Zum zweiten Mal fand ein Zusatztraining im Vorfeld dieses Auswahlverfahrens statt. J. Reinhold und L. Sauermann ( $U$ Stanford) betreuten in der zweiten Jahreshälfte 2015 per E-Mail-Korrespondenz sehr intensiv anfangs acht, dann sechs Schüler, die schon an IMOVorbereitungslehrgängen teilgenommen hatten und die auch noch in diesem Jahr startberechtigt waren. Vier dieser Schüler konnten sich letztendlich für die IMO 2016 qualifizieren.

Vom 15.-18. Juni fand ein Lehrgang an der JacobsUniversity Bremen statt, bei dem auch ausländische Mentoren ihre langiährigen Trainingserfahrungen aus Russland, Weißrussland, Thailand und dem Iran mit einbrachten. Zudem führte das Team vom 25.-29. Juni ein selbstständig organisiertes Intensivtraining in einer Jugendherberge in Erfurt durch.

Seit 2007 gibt es das Programm ,Jugend trainiert Mathematik“ (JuMa). Es wurde u. a. zur besseren Vorbereitung unserer Schülerinnen und Schüler auf die IMO initiiert. Viele der erfolgreichen Teilnehmer an den bundesweiten Mathematik-Wettbewerben und auch fünf unserer sechs IMO-Teilnehmer wurden durch dieses Projekt gefördert.

Die gesamte organisatorische Vorbereitung und Durchführung der Klausuren, der Seminare und der Reise wurden wiederum von der Geschäftsstelle der bundesweiten Mathematik-Wettbewerbe unter Leitung von H.-H. Langmann in gewohnt perfekter Weise abgewickelt. Allen, die an der Organisation und der Vorbereitung des deutschen Teams beteiligt waren, gebührt herzlicher Dank.

\section{2}

\section{Der Ablauf der 57. IMO}

Seit 2005 wird die erste IMO-Klausur üblicherweise bereits am zweiten Tag nach Anreise geschrieben. Vergangenes Jahr hatten die Schüler die Zeitumstellung von sechs Stunden am ersten Klausurtag noch nicht richtig überwunden. Daher reiste der stellvertretende Delegations- 
leiter zusammen mit den Schülern dieses Jahr bereits einen Tag früher an.

Die Eröffnungsfeier am 10. Juli im Queen Elizabeth Stadium wurde effektvoll umrahmt von Musik, die der Hongkonger Komponist Dr. Mui Kwong Chiu extra für diese IMO geschaffen hat. Zur traditionellen Parade kamen wieder alle teilnehmenden Mannschaften auf die Bühne, diesmal in der Reihenfolge, in der die Länder zum ersten Mal an der IMO teilnahmen.

Am II. und 12. Juli wurden vormittags die beiden viereinhalbstündigen Klausuren geschrieben. Die Arbeitsbedingungen waren sehr gut. Die Verständnisfragen, die die Schüler in der ersten halben Stunde der Klausur schriftlich stellen konnten, wurden wieder elektronisch an die Jury übermittelt. Dort wurden vom jeweiligen Delegationsleiter die Antworten formuliert und nach Genehmigung durch die Jury wieder elektronisch an die Schüler zurückgeschickt. Da Standardantworten schon vorformuliert waren, konnten innerhalb kurzer Zeit 85 Fragen am ersten Tag und 95 Fragen am zweiten Tag beantwortet werden. Die deutschen Schüler stellten insgesamt vier Fragen.

Nach der Durchsicht der Schülerlösungen durch die Delegationsleitungen fand vom 13.-14. Juli die endgültige Festlegung der Bewertung mit den Koordinatoren statt. Hierzu hatten die Veranstalter ein Team von ca. 62 einheimischen und 16 internationalen Experten zusammengestellt. Aus Deutschland waren als eingeladene Koordinatoren wieder Stephan Neupert, zurzeit Doktorand an der LMU München, und Lisa Sauermann, zurzeit PhDStudentin an der Stanford University, beteiligt. Die gesamte Koordination verlief sehr fair und war gut organisiert.

Am Vormittag des letzten Tages hielt der FieldsMedaillen-Preisträger Efim Zelmanov die inzwischen zur Tradition gewordene „IMO Lecture“. Die Schüler waren begeistert von dieser spannenden und verständlichen Vorlesung über kombinatorische Fragestellungen.

Die Preisverleihung fand am I5. Juli im Hong Kong Convention and Exhibition Centre statt. Die Medaillengewinner, in Gruppen auf die Bühne gerufen, kamen mit den Fahnen ihrer Heimatländer, sodass auf der Bühne eine bunte Fahnenreihe im Blitzlichtgewitter zu sehen war. Besonders gefeiert wurde die Mannschaft aus Hongkong, die in diesem Jahr mit drei Gold-, zwei Silber- und einer Bronzemedaille erstmalig seit Beginn ihrer IMOTeilnahme 1988 mit Platz 9 zu den zehn besten Ländern gehört. Zum Abschluss wurde die IMO-Fahne feierlich an den nächsten Veranstalter Brasilien übergeben und mit einem Film über Rio de Janeiro und die IMO 2017 für das nächste Jahr eingeladen. Im Anschluss an die Preisverleihung gab es eine Farewell Party mit einem beeindruckenden Bühnenprogramm.
An der 57. IMO nahmen 109 Länder mit 602 Schülerinnen und Schülern teil (vgl. die Ergebnisübersicht auf Seite 222).

Von den 104 Ländern, die an der IMO 2015 in Thailand teilgenommen hatten, fehlten dieses Jahr Bolivien, Kuba und Panama. In diesem Jahr nahmen sieben Länder erstmalig an einer IMO teil: Ägypten, Irak, Jamaika, Kenia, Laos, Madagaskar und Myanmar. Außerdem nahm nach zweijähriger Pause Honduras wieder teil.

Die internationale Jury, bestehend aus den 109 Delegationsleitern und einem Chairman des veranstaltenden Landes, begann am Morgen des 7. Juli mit ihrer Arbeit. Als Chairman fungierte Prof. Dr. Kar Ping Shum. Die meisten Jury-Sitzungen leitete aber Andy Loo, der 2012 auf der IMO in Argentinien für Hongkong eine Silbermedaille erringen konnte. Erstmalig wurde in der Jury elektronisch abgestimmt. Dies stellte sich als sehr effizient heraus, da die Ergebnisse sofort auf dem Bildschirm sichtbar waren. Ein Novum waren damit auch geheime Abstimmungen.

Jedes teilnehmende Land hat das Recht, Aufgabenvorschläge einzureichen. In diesem Jahr wurden den Veranstaltern 121 Aufgaben aus 40 Ländern zugesandt. Eine Aufgabenkommission des Veranstalters (Problem Selection Committee, PSC), bestehend aus elf Mitgliedern, stellte daraus im Vorfeld der IMO 32 Aufgaben - je acht aus den Gebieten Algebra, Kombinatorik, Geometrie und Zahlentheorie - für eine Shortlist zusammen, welche die Grundlage für die Auswahl der Jury bildeten. Im Gegensatz zu den Vorjahren bestand das PSC in diesem Jahr nur aus einheimischen Mitgliedern.

Die Jury legte nach intensiven Diskussionen sechs dieser Aufgaben für die beiden Klausuren fest, wobei die Aufgaben einerseits eine gute Mischung von unterschiedlichen Schwierigkeitsgraden und mathematischen Gebieten sein sollen, andererseits aber auch möglichst keine „Standard“-Lösungen zulassen sollen. Zu Beginn der Auswahl konnte wieder jedes Jury-Mitglied in einem „Beauty Contest" die Eleganz und den Schwierigkeitsgrad persönlich bewerten. Die summarischen Ergebnisse sind äußerst hilfreich bei der anschließenden, oft sehr kontroversen Meinungsbildung. Aufgaben aus Deutschland waren in diesem Jahr leider nicht dabei.

Nach der Auswahl der Aufgaben wurde zunächst eine finale englische Version erstellt und diese dann in die vier anderen offiziellen Sprachen Deutsch, Französisch, Russisch und Spanisch übersetzt und von der Jury bestätigt. Jeder Schüler und jede Schülerin erhält die Aufgaben in der Muttersprache und einer zweiten Sprache eigener Wahl. Demgemäß übersetzten die entsprechenden Delegationsleiter die Aufgabentexte in die restlichen 53 Sprachen. Auch alle diese Versionen wurden nach Prüfung durch die Jury bestätigt. Insgesamt standen die Auf- 


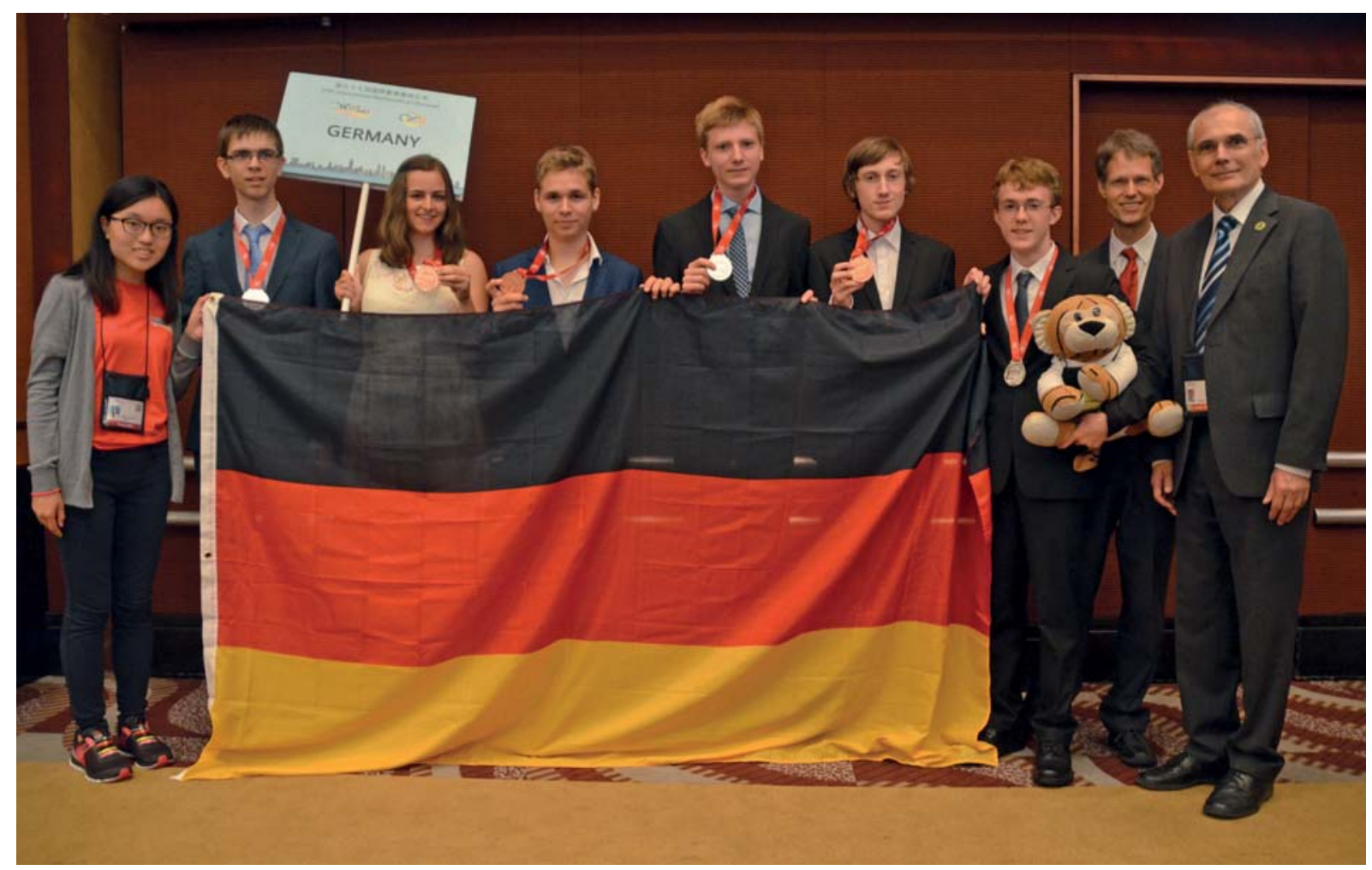

V. I. n. r.: Vivian Chung (Guide), Sebastian Meyer, Susanne Armbruster, Branko Juran, Martin Drees, Manfred Paul, Ferdinand Wagner, Dr. Eric Müller, Prof. Dr. Jürgen Prestin (Foto: Privat)

gaben in 58 Sprachversionen zur Verfügung und sind auf www.imo-official.org verfügbar.

Bei der Bewertung der Lösungen wurden 35,2\% der möglichen Punkte vergeben. Diese IMO lag damit genau im Durchschnitt der letzten 10 Jahre. Die beiden Extrema in diesen 10 Jahren waren 2014 mit $38,2 \%$ und 2015 mit $30,9 \%$.

In diesem Jahr erreichten sechs Schüler die volle Punktzahl (drei aus der Republik Korea, zwei aus den USA sowie einer aus der VR China). In der „Hall of Fame“ aller IMO-Teilnehmer seit 1959 (www. Mathematik-Olympiaden.de bzw. www.imo-official.org/ hall.aspx) gab es an der Spitze keine Veränderungen. In dieser Liste liegen unverändert Lisa Sauermann auf Platz 3, Christian Reiher auf Platz 5, Wolfgang Burmeister auf Platz 7, Martin Härterich auf Platz 10 und Peter Scholze auf Platz II. Im exklusiven „Club der IMO-Teilnehmer mit mindestens 3 Goldmedaillen“ gibt es drei neue Mitglieder: Kevin Sun aus Kanada, Sheldon Kieren Tan aus Singapur und Allen Liu aus den USA.

Das Reglement, das seit vielen Jahren festgeschrieben ist, sieht vor, dass nicht mehr als die Hälfte der Teilnehmer einen Preis erhält und dass die Anzahlen der I., 2. bzw. 3. Preise möglichst das Verhältnis $I: 2: 3$ aufweisen sollten. Die diesjährigen Punktgrenzen sind in Tabelle 2 angegeben. Eine spannende Frage bleibt immer, ob die Jury das Reglement streng auslegt oder eher zu approximativen Varianten neigt. Nach 2015 hat die Jury zum zweiten Mal jeweils nur über höhere und niedrigere Wahlen von Punktegrenzen für die Preise abgestimmt und erfuhr erst am Ende die resultierenden konkreten Punktezahlen. Anders als oft in den Vorjahren, aber wie im letzten Jahr, hat sich die Jury dann bei den Preisgrenzen für die kleinere Medaillenanzahl entschieden.

Es gab auch in diesem Jahr keinen Sonderpreis für die besonders elegante Lösung einer Aufgabe.

Tabelle 2. Die Punktgrenzen für die Preise

\footnotetext{
44 Goldmedaillen für $\geq 29$ Punkte (von 42)

I0I Silbermedaillen für $\geq 22$ Punkte

I35 Bronzemedaillen für $\geq 16$ Punkte

280 Medaillen bei 602 Teilnehmern
}

\section{Die deutsche IMO-Mannschaft}

Sehr gefreut haben wir uns, dass alle sechs deutschen Teilnehmer eine Medaille erringen konnten (Tabelle 3). In der inoffiziellen Länderwertung liegen wir auf Rang 19, nach Rang 27 auf der letztjährigen IMO in Thailand. In den Jahren davor erzielten wir 2014 in Südafrika Rang 16 und 2013 in Kolumbien Rang 27. 
Tabelle 3. Die Ergebnisse des deutschen Teams

\begin{tabular}{lcl}
\hline Name & Punkte & Preis \\
\hline Martin Drees & 27 & Silber \\
Sebastian Meyer & 25 & Silber \\
Ferdinand Wagner & 23 & Silber \\
Manfred Paul & 21 & Bronze \\
Branko Juran & 19 & Bronze \\
Susanne Armbruster & 16 & Bronze \\
\hline
\end{tabular}

Tabelle 4. Die Ergebnisse der einzelnen Aufgaben (in \%)

\begin{tabular}{clrrc}
\hline Aufgabe & Gebiet & Alle & Top 10 & Deutsches Team \\
\hline 1 & Geometrie & 75,3 & 96,2 & 95,2 \\
2 & Kombinatorik & 29,0 & 84,5 & 69,0 \\
3 & Zahlentheorie & 3,6 & 25,2 & 16,7 \\
4 & Zahlentheorie & 67,8 & 97,9 & 100,0 \\
5 & Algebra & 24,0 & 80,5 & 23,8 \\
6 & Kombinatorik & 11,5 & 46,9 & 7,1 \\
Alle & & 35,2 & 71,9 & 52,0 \\
\hline
\end{tabular}

Unser Team enthielt die beiden Abiturienten Susanne Armbruster und Ferdinand Wagner, der an insgesamt drei IMOs teilnahm. Die anderen vier Teilnehmer können sich wieder für die IMO 2017 in Rio de Janeiro qualifizieren.

Interessant ist ein Blick auf die Ergebnisse bei den einzelnen Aufgaben. Der Vergleich der erreichten Resultate (in Prozent) aller IMO-Teilnehmer, der Schüler der besten zehn Teams sowie des deutschen Teams gibt Aufschluss darüber, wie unsere Schüler die Aufgaben relativ bewältigten (Tabelle 4). Bemerkenswert in diesem Jahr war das sehr gute Abschneiden unseres Teams in Geometrie und Zahlentheorie.

\section{IMO Advisory Board}

In diesem Jahr fanden turnusgemäß Wahlen zum IMO Advisory Board statt. Die neue Zusammensetzung dieses Gremiums ist in Tabelle 5 angegeben.
Die seit mehreren Jahren arbeitende Ethik-Kommission unter Leitung von Prof. Dr. Rafael Sánchez aus Venezuela, die sich mit Ehrlichkeit und Fairness der Olympiaden befassen soll, musste in diesem Jahr den Fall eines ehemaligen Teilnehmers behandeln, der in seinem heutigen Lebenslauf für das Jahr der IMO-Teilnahme „Studium“ angibt. Es wurde beschlossen, diese IMO-Teilnahme aus den Ergebnislisten zu streichen.

\section{Ausblick und IMO-Informationen}

Die nächte IMO wird vom 12.-23. Juli 2017 in Rio de Janeiro, Brasilien, stattfinden. Austragungsland der IMO 2018 ist Rumänien und 2019 trägt das Vereinigte Königreich die IMO aus. In diesem Jahr bestätigte die Jury die Veranstalter für 2020 (Russland) und 202I (USA). Interessensbekundungen für 2022 und 2023 liegen aus Norwegen und Japan vor.

Für weitere Informationen zu mathematischen Schülerwettbewerben verweisen wir auf die Webseite www.mathe-wettbewerbe.de.

Speziell zu den IMOs sind folgende Webseiten empfehlenswert: www.imo-official.org

www.mathematik-olympiaden.de/IMOs/imo.html

Prof. Dr. Jürgen Prestin, Universität zu Lübeck, Institut für Mathematik, Ratzeburger Allee 160, 23562 Lübeck prestin@math.uni-luebeck.de

Jürgen Prestin ist seit 2000 Inhaber einer Professur für Mathematik an der Universität zu Lübeck. Seine Forschungsschwerpunkte liegen in Approximationstheorie und Fourier-Analysis. Seit 2010 ist er I. Vorsitzender des MathematikOlympiaden e. V. und seit 2015 Delegationsleiter der deutschen IMO-Mannschaft.

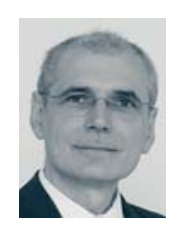

Tabelle 5. Die Mitglieder des IMO Advisory Boards

\begin{tabular}{|c|c|c|c|}
\hline Funktion & Name & Land & Amtszeit \\
\hline Vorsitzender & Geoff Smith & Vereinigtes Königreich & bis 2018 \\
\hline Sekretär & Gregor Dolinar & Slowenien & bis 2020 \\
\hline Mitglied & Nazar Agakhanov & Russland & bis 2018 \\
\hline Mitglied & Dávid Kunszenti-Kovács & Norwegen & bis 2020 \\
\hline Mitglied & Yongjin Song & Südkorea & bis 2018 \\
\hline ex officio IMO 2016 & Kar Ping Shum & Hongkong & bis 2017 \\
\hline ex officio IMO 2017 & Edmilson Luis Rodrigues Motta & Brasilien & bis 2018 \\
\hline ex officio IMO 2018 & Radu Gologan & Rumänien & bis 2019 \\
\hline
\end{tabular}




\section{Tag}

I. Das Dreieck $B C F$ habe einen rechten Winkel in $B$. Es sei $A$ der Punkt auf der Geraden $C F$, für den $F A=F B$ gilt und $F$ zwischen $A$ und $C$ liegt. Der Punkt $D$ sei so gewählt, dass $D A=D C$ gilt und $A C$ den Winkel $\Varangle D A B$ halbiert. Der Punkt $E$ sei so gewählt, dass $E A=E D$ gilt und $A D$ den Winkel $\Varangle E A C$ halbiert. Es sei $M$ der Mittelpunkt von $C F$. Ferner sei $X$ derjenige Punkt, für den $A M X E$ ein Parallelogramm (mit $A M \| E X$ und $A E \| M X$ ) ist.

Man beweise, dass sich $B D, F X$ und $M E$ in einem Punkt schneiden.

(Belgien)

2. Bestimme alle positiven ganzen Zahlen $n$, für die jedes Feld einer $n \times n$ Tabelle so mit einem der Buchstaben I, $M$ und $O$ gefüllt werden kann, dass:

- in jeder Zeile und in jeder Spalte ein Drittel der Einträge $I$, ein Drittel $M$ und ein Drittel $O$ sind, und

- in jeder Diagonale, in der die Anzahl der Einträge ein Vielfaches von drei ist, ein Drittel der Einträge I, ein Drittel $M$ und ein Drittel $O$ sind.

Bemerkung: Die Zeilen und Spalten der $n \times n$ Tabelle sind in üblicher Reihenfolge von 1 bis $n$ nummeriert. Damit entspricht jedes Feld einem Paar positiver ganzer Zahlen $(i, j)$ mit $1 \leqslant i, j \leqslant n$. Für $n>1$ hat die Tabelle $4 n-2$ Diagonalen, die sich in zwei Arten aufteilen. Eine Diagonale der ersten Art besteht aus allen Feldern $(i, j)$, für die $i+j$ eine Konstante ist. Eine Diagonale der zweiten Art besteht aus allen Feldern $(i, j)$, für die $i-j$ eine Konstante ist.

(Australien)

3. Es sei $P=A_{1} A_{2} \ldots A_{k}$ ein ebenes konvexes Vieleck. Die Eckpunkte $A_{1}, A_{2}, \ldots, A_{k}$ haben ganzzahlige Koordinaten und liegen auf einem Kreis. Es sei $S$ der Flächeninhalt von $P$. Ferner sei eine ungerade positive ganze Zahl $n$ gegeben, sodass die Quadrate der Seitenlängen von $P$ durch $n$ teilbare ganze Zahlen sind.

Man beweise, dass $2 S$ eine durch $n$ teilbare ganze Zahl ist.

(Russland)

Arbeitszeit: 41/2 Stunden an jedem Tag. Bei jeder Aufgabe waren 7 Punkte erreichbar.

Erklärend zu Aufgabe 4 sei angefügt, dass Hongkong übersetzt „duftender Hafen“ bedeutet. Im englischen Original der Aufgabe 6 heißt die Person Geoff (vgl. Tabelle 5). In vielen Übersetzungen wurde der Name passend geändert.

\section{Tag}

4. Eine Menge von positiven ganzen Zahlen heiße duftend, wenn sie mindestens zwei Elemente enthält und jedes ihrer Elemente mit wenigstens einem anderen ihrer Elemente mindestens einen Primfaktor gemeinsam hat. Es sei $P(n)=n^{2}+n+1$. Man bestimme die kleinstmögliche positive ganze Zahl $b$, für die eine nicht-negative ganze Zahl a existiert, sodass die Menge

$$
\{P(a+1), P(a+2), \ldots, P(a+b)\}
$$

duftend ist.

(Luxemburg)

\section{Die Gleichung}

$(x-1)(x-2) \cdots(x-2016)=(x-1)(x-2) \cdots(x-2016)$,

mit 2016 Linearfaktoren auf jeder Seite, steht auf einer Tafel. Man bestimme das kleinstmögliche $k$, für das genau k dieser 4032 Linearfaktoren gelöscht werden können, sodass auf jeder Seite mindestens ein Linearfaktor verbleibt und die entstehende Gleichung keine reelle Lösung besitzt.

(Russland)

6. In der Ebene seien $n \geqslant 2$ Strecken so gegeben, dass sich je zwei Strecken kreuzen und keine drei durch einen gemeinsamen Punkt verlaufen. Lisa soll von jeder Strecke einen ihrer Endpunkte auswählen und dort einen Frosch so hinsetzen, dass er zum anderen Endpunkt blickt. Dann wird sie $n-1 \mathrm{mal}$ in die Hände klatschen. Jedes Mal, wenn sie klatscht, springt jeder Frosch sofort vorwärts auf den nächsten Schnittpunkt auf seiner Strecke. Die Frösche wechseln nie die Sprungrichtung. Lisa möchte die Frösche so hinsetzen, dass sich niemals zwei Frösche gleichzeitig auf dem gleichen Schnittpunkt befinden.

(a) Man beweise, dass Lisa dies immer erreichen kann, wenn $n$ ungerade ist.

(b) Man beweise, dass Lisa dies niemals erreichen kann, wenn $n$ gerade ist.

(Tschechische Republik)

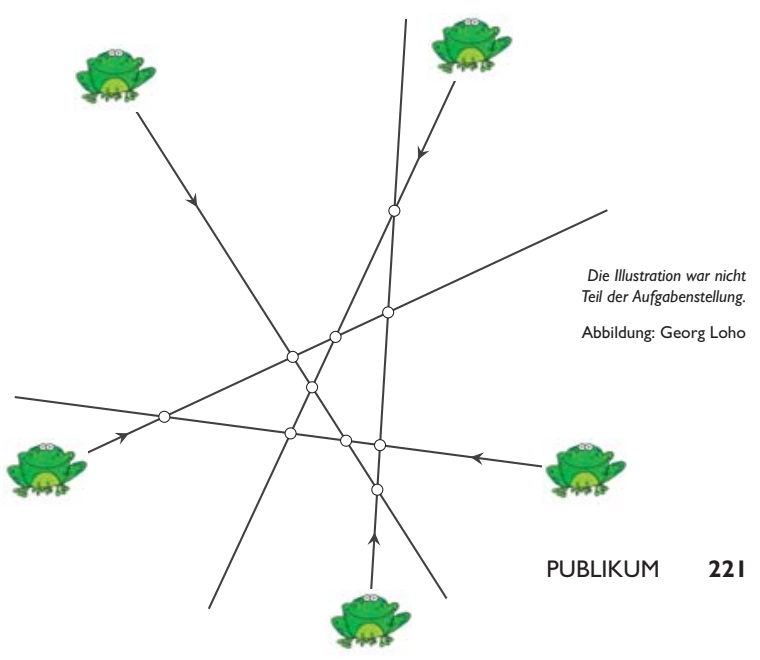


57. IMO 2016 - Länderübersicht (inoffiziell)

\begin{tabular}{|c|c|c|c|c|c|}
\hline $\mathrm{N}$ & Land & $P$ & G & $\mathrm{S}$ & B \\
\hline I & USA & 214 & 6 & - & - \\
\hline 2 & Republik Korea & 207 & 4 & 2 & - \\
\hline 3 & Volksrepublik China & 204 & 4 & 2 & - \\
\hline 4 & Singapur & 196 & 4 & 2 & - \\
\hline 5 & Taiwan & 175 & 3 & 3 & - \\
\hline 6 & Demokratische Volksrepublik Korea & 168 & 2 & 4 & - \\
\hline \multirow[t]{2}{*}{7} & Russland & 165 & 4 & I & I \\
\hline & Vereinigtes Königreich & 165 & 2 & 4 & - \\
\hline 9 & Hongkong & 161 & 3 & 2 & I \\
\hline 10 & Japan & 156 & I & 4 & I \\
\hline II & Vietnam & $|5|$ & I & 4 & I \\
\hline \multirow[t]{2}{*}{12} & Kanada & 148 & 2 & 2 & I \\
\hline & Thailand & 148 & 2 & 2 & I \\
\hline 14 & Ungarn & 145 & I & 3 & 2 \\
\hline \multirow[t]{2}{*}{15} & Brasilien & 138 & - & 5 & I \\
\hline & Italien & 138 & I & 3 & - \\
\hline 17 & Philippinen & 133 & 2 & 2 & - \\
\hline 18 & Bulgarien & 132 & - & 3 & 3 \\
\hline 19 & Deutschland & $|3|$ & - & 3 & 3 \\
\hline \multirow[t]{2}{*}{20} & Indonesien & 130 & - & 3 & 3 \\
\hline & Rumänien & 130 & - & 5 & I \\
\hline 22 & Israel & 127 & - & 3 & 3 \\
\hline 23 & Mexiko & 126 & - & 4 & I \\
\hline 24 & Islamische Republik Iran & 125 & - & 3 & 3 \\
\hline \multirow[t]{3}{*}{25} & Australien & 124 & - & 2 & 4 \\
\hline & Frankreich & 124 & - & 3 & 2 \\
\hline & Peru & 124 & - & 2 & 3 \\
\hline 28 & Kasachstan & 122 & I & I & 3 \\
\hline 29 & Türkei & 121 & - & 2 & 4 \\
\hline \multirow[t]{3}{*}{30} & Armenien & 118 & - & I & 4 \\
\hline & Kroatien & 118 & - & I & 4 \\
\hline & Ukraine & 118 & - & 2 & 4 \\
\hline 33 & Mongolei & 115 & - & 2 & 2 \\
\hline 34 & Indien & 113 & - & I & 5 \\
\hline \multirow[t]{2}{*}{35} & Bangladesch & 112 & - & I & 3 \\
\hline & Weißrussland & 112 & - & I & 4 \\
\hline \multirow[t]{2}{*}{37} & Schweden & 109 & - & 3 & - \\
\hline & Tschechische Republik & 109 & - & 2 & I \\
\hline 39 & Macao & 108 & I & I & - \\
\hline 40 & Serbien & 106 & - & I & 4 \\
\hline 41 & Saudi-Arabien & 104 & - & - & 4 \\
\hline 42 & Polen & 102 & - & 2 & 2 \\
\hline 43 & Schweiz & 99 & - & I & 4 \\
\hline 44 & Niederlande & 98 & - & - & 3 \\
\hline 45 & Bosnien und Herzegowina & 97 & - & - & 4 \\
\hline 46 & Österreich & 89 & - & - & 3 \\
\hline 47 & Portugal & 88 & - & - & I \\
\hline 48 & Syrien & 87 & - & - & 3 \\
\hline 49 & Spanien & 86 & - & - & 2 \\
\hline \multirow[t]{2}{*}{50} & Griechenland & 84 & - & - & 2 \\
\hline & Litauen & 84 & - & - & 3 \\
\hline 52 & Belgien & 82 & - & - & 3 \\
\hline 53 & Neuseeland & 81 & - & I & I \\
\hline 54 & Aserbaidschan & 79 & - & - & I \\
\hline 55 & Slowakei & 78 & - & - & 2 \\
\hline
\end{tabular}

\begin{tabular}{|c|c|c|c|c|c|}
\hline $\mathrm{N}$ & Land & $P$ & G & $S$ & B \\
\hline 56 & Malaysia & 77 & - & - & 2 \\
\hline 57 & Argentinien & 75 & - & - & 2 \\
\hline 58 & Südafrika & 73 & - & - & I \\
\hline \multirow[t]{2}{*}{59} & Costa Rica & 69 & - & - & 2 \\
\hline & Georgien & 69 & - & - & I \\
\hline 61 & Estland & 67 & - & - & I \\
\hline 62 & Tadschikistan & 66 & - & - & - \\
\hline \multirow[t]{3}{*}{63} & Moldawien & 65 & - & - & I \\
\hline & Republik Zypern & 65 & - & I & - \\
\hline & Slowenien & 65 & - & - & - \\
\hline \multirow[t]{2}{*}{66} & Kolumbien & 63 & - & - & 2 \\
\hline & Sri Lanka & 63 & - & - & I \\
\hline 68 & El Salvador (5) & 60 & - & - & I \\
\hline \multirow[t]{2}{*}{69} & Albanien & 58 & - & - & I \\
\hline & Turkmenistan & 58 & - & - & - \\
\hline \multirow[t]{2}{*}{71} & Finnland & 55 & - & - & - \\
\hline & Paraguay & 55 & - & - & 2 \\
\hline 73 & Ehem. Jug. Rep. Mazedonien & 53 & - & - & - \\
\hline 74 & Lettland & 52 & - & - & - \\
\hline 75 & Irland & 51 & - & - & - \\
\hline 76 & Tunesien & 50 & - & - & - \\
\hline \multirow[t]{2}{*}{77} & Kosovo & 47 & - & - & I \\
\hline & Usbekistan & 47 & - & - & I \\
\hline 79 & Marokko & 46 & - & - & I \\
\hline 80 & Nicaragua (5) & 45 & - & - & I \\
\hline 81 & Dänemark & 44 & - & - & - \\
\hline 82 & Algerien (4) & 41 & - & - & - \\
\hline 83 & Ecuador & 38 & - & - & - \\
\hline \multirow[t]{2}{*}{84} & Kirgisistan & 34 & - & - & - \\
\hline & Norwegen & 34 & - & - & - \\
\hline 86 & Venezuela (3) & 29 & - & - & I \\
\hline 87 & Puerto Rico (2) & 27 & - & - & I \\
\hline \multirow[t]{2}{*}{88} & Montenegro (2) & 24 & - & I & - \\
\hline & Nigeria & 24 & - & - & - \\
\hline 90 & Island & 23 & - & - & - \\
\hline \multirow[t]{2}{*}{91} & Chile (3) & 18 & - & - & - \\
\hline & Pakistan & 18 & - & - & - \\
\hline 93 & Uruguay (I) & 17 & - & - & I \\
\hline 94 & Trinidad und Tobago (4) & 15 & - & - & - \\
\hline 95 & Luxemburg (3) & 14 & - & - & - \\
\hline \multirow[t]{2}{*}{96} & Kambodscha & 13 & - & - & - \\
\hline & Myanmar & 13 & - & - & - \\
\hline 98 & Uganda & 12 & - & - & - \\
\hline 99 & Kenia & II & - & - & - \\
\hline \multirow[t]{2}{*}{100} & Honduras (2) & 10 & - & - & - \\
\hline & Madagaskar (5) & 10 & - & - & - \\
\hline 102 & Jamaika (I) & 9 & - & - & - \\
\hline 103 & Botswana & 7 & - & - & - \\
\hline \multirow[t]{2}{*}{104} & Ghana (3) & 5 & - & - & - \\
\hline & Ägypten (5) & 5 & - & - & - \\
\hline 106 & Tansania (2) & 3 & - & - & - \\
\hline \multirow[t]{2}{*}{107} & Irak (5) & 2 & - & - & - \\
\hline & Liechtenstein (I) & 2 & - & - & - \\
\hline 109 & Laos & 0 & - & - & - \\
\hline
\end{tabular}

Legende: N - Platzierung, P - Punktzahl, G - Anzahl der Goldmedaillen, S - Anzahl der Silbermedaillen, B - Anzahl der Bronzemedaillen. Jede Mannschaft bestand aus sechs bzw. der in Klammern angegebenen Anzahl von Schülern. Eine vollständige Mannschaft (sechs Schüler) konnte maximal 252 Punkte erreichen. 